\title{
Filtering-Based Fault Detection for Stochastic Markovian Jump System with Distributed Time-Varying Delays and Mixed Modes
}

\author{
Yucai Ding, ${ }^{1}$ Hong Zhu, ${ }^{1}$ Shouming Zhong, ${ }^{2}$ \\ Yuping Zhang, ${ }^{1}$ and Jianwei $\mathrm{Xia}^{3}$ \\ ${ }^{1}$ School of Automation Engineering, University of Electronic Science and Technology of China, \\ Chengdu 611731, China \\ ${ }^{2}$ School of Mathematical Sciences, University of Electronic Science and Technology of China, \\ Chengdu 611731, China \\ 3 School of Mathematics Science, Liaocheng University, Liaocheng 252000, China
}

Correspondence should be addressed to Yucai Ding, dycyer@163.com

Received 10 May 2012; Revised 10 November 2012; Accepted 13 November 2012

Academic Editor: Jong Hae Kim

Copyright (C) 2012 Yucai Ding et al. This is an open access article distributed under the Creative Commons Attribution License, which permits unrestricted use, distribution, and reproduction in any medium, provided the original work is properly cited.

The problem of fault detection for stochastic Markovian jump system is considered. The system under consideration involves discrete and distributed time-varying delays, Itô-type stochastic disturbance, and different system and delay modes. The aim of this paper is to design a fault detection filter such that the fault detection system is stochastically stable and satisfies a prescribed $H_{\infty}$ disturbance attenuation level. By using a novel Lyapunov functional, a mix-mode-dependent sufficient condition is formulated in terms of linear matrix inequalities. A numerical example is given to illustrate the effectiveness of the proposed main results.

\section{Introduction}

Fault detection received considerable attention over the past decades because of the increasing demand for higher performance, safety, and reliability standards. In recently, many effective methods have been developed for fault detection. To the best of the authors' knowledge, the published results can be categorized into three approaches. The first category is the filter- or observer-based approaches, where filters are used to generate residual signals to detect and estimate the fault, for example, [1-9]. In the fault detection scheme based on filter or observer, a fault cannot only be detected but also be approximated, and the 
fault estimate can be further used in fault-tolerant control. The second category is the statistic approach, where the Bayesian theory and likelihood method are used to evaluate the fault signals [10]. The third category is the geometric approach. By utilizing the geometric framework, a set of residuals is generated such that each residual is affected by one fault and is partially decoupled from others [11]. In the framework of fault detection, faults are detected by setting a predefined threshold on residual signals. Once the value of residual evaluation function excesses the predefined threshold, an alarm of faults is generated. For example, by using Luenberger type observers, the authors of $[6,12]$ present an explicit expression of the filters for the fault such that both asymptotic stability and a prescribed level of disturbance attenuation are satisfied for all admissible nonlinear perturbations; by using the measured output probability density functions (PDFs), the authors of $[13,14]$ construct a stable filter-based residual generator.

Markovian jump systems (MJSs) are a special class of switched systems. The state vector of such system has two components $x(t)$ and $r(t)$. The first one is in general referred to as the state, and the second one is regarded as the mode. In its operation, the jump system will switch from one mode to another in a random way, based on a Markovian chain with finite state space. These systems are very common in economic systems, communication systems, robot manipulator systems and circuit systems, and so forth. Time delay is an inherent characteristic of many physical systems, which occurs due to signal transmission, inevitable defects of control equipment, and so on. The systems with or without time delays are convergent when time delays are close to zero. otherwise, they may be divergent. In other words, time delays, either constant or time varying, can degrade the performance of systems designed without considering the delays and can even destabilize the systems. Due to their extensive practical applications, considerable attention has been devoted to MJSs, see, for example, [15-18] for stability, [18-25] for control, and [25-32] for state estimation. More recently, the methods of fault estimation and fault detection have been extended successfully to MJSs [33-40]. From the published results, the delay mode is assumed to be the same as the system matrices mode. However, the assumption cannot always be satisfied in real applications. In some practical systems, variations of delay usually depend on phenomena which may not cause abrupt changes in other system parameters. For instance, in networked control systems, the randomness of delay is a result of communication network issues, but the process itself may contain separate sources of randomness which means that the system matrices mode may be different with the delay mode [41]. Therefore, it is important and necessary to pay attention to the study of Markovian jump systems with different system and delay modes. Furthermore, it appears that general results pertaining to fault detection for stochastic MJSs with discrete and distributed time delays, Itô-type stochastic disturbance and different system and delay modes are few and restricted, despite its practical importance, mainly due to the mathematical difficulties in dealing with such mixed modes. Research in this area should be interesting yet challenging as it involves the combination of two different jumping modes, which has motivated this paper.

This paper deals with the problem of fault detection for stochastic MJSs with discrete and distributed time-varying delays, Itô-type stochastic disturbance, and different system and delay modes. By using a novel mix-mode-dependent Lyapunov functional, a new sufficient condition on stochastic stability with an $H_{\infty}$ performance is derived in terms of linear matrix inequalities (LMIs). Based on this, the existence condition of the fault detection filter which guarantees stochastic stability and the $H_{\infty}$ performance of the corresponding augmented system is presented. A numerical example is provided to show the effectiveness of the proposed results. 
Notation. Throughout this paper, $\mathbb{R}^{n}$ denotes the $n$ dimensional Euclidean space. $\lambda_{\max }(Q)$ and $\lambda_{\min }(Q)$ denote, respectively, the maximal and minimal eigenvalue of matrix $Q . E\{\cdot\}$ refers to the expectation operator with respect to some probability measure $D$. We use $\operatorname{diag}\{\cdot, \cdot, \cdot\}$ as a block-diagonal matrix. $A>0(<0)$ means that $A$ is a symmetric positive (negative) definite matrix, $A^{-1}$ denotes the inverse of matrix $A$. $A^{T}$ denotes the transpose of matrix $A$, and $I$ is the identity matrix with compatible dimension.

\section{System Description and Definitions}

Consider the following stochastic MJS with mode-dependent time-varying delays:

$$
\begin{aligned}
& \mathrm{d} x(t)=[ A\left(r_{t}\right) x(t)+A_{1}\left(r_{t}\right) x\left(t-\tau\left(t, s_{t}\right)\right)+A_{2}\left(r_{t}\right) \int_{t-\tau\left(t, s_{t}\right)}^{t} x(s) \mathrm{d} s \\
&\left.+B_{0}\left(r_{t}\right) u(t)+B_{1}\left(r_{t}\right) v(t)+B_{2}\left(r_{t}\right) f(t)\right] \mathrm{d} t+G_{1}\left(r_{t}\right) x(t) \mathrm{d} \omega(t), \\
& \mathrm{d} y(t)= {\left[C\left(r_{t}\right) x(t)+C_{1}\left(r_{t}\right) x\left(t-\tau\left(t, s_{t}\right)\right)+C_{2}\left(r_{t}\right) \int_{t-\tau\left(t, s_{t}\right)}^{t} x(s) \mathrm{d} s\right.} \\
&\left.+D_{1}\left(r_{t}\right) v(t)+D_{2}\left(r_{t}\right) f(t)\right] \mathrm{d} t+G_{2}\left(r_{t}\right) x(t) \mathrm{d} \omega(t), \\
& x(t)=\phi(t), \quad t \in[-\tau, 0]
\end{aligned}
$$

where $x(t) \in \mathbb{R}^{n}$ is the state vector; $u(t)$ is the exogenous disturbance input which belongs to $L_{2}[0 \infty) ; v(t)$ the unknown input; $f(t)$ is the fault to be detected; $y(t) \in \mathbb{R}^{p}$ is the measured output; $\omega(t)$ is a zero-mean one-dimensional Wiener process satisfying $E\{\omega(t)\}=0$ and $E\left\{\omega^{2}(t)\right\}=t ; \phi(t)$ is a compatible vector-valued initial function defined on $[-\tau, 0] ; A\left(r_{t}\right)$, $A_{1}\left(r_{t}\right), A_{2}\left(r_{t}\right), B_{0}\left(r_{t}\right), B_{1}\left(r_{t}\right), B_{2}\left(r_{t}\right), G_{1}\left(r_{t}\right), C\left(r_{t}\right), C_{1}\left(r_{t}\right), C_{2}\left(r_{t}\right), D_{1}\left(r_{t}\right), D_{2}\left(r_{t}\right)$, and $G_{2}\left(r_{t}\right)$ are real constant matrices with appropriate dimensions. $\tau\left(t, s_{t}\right)$ is the mode-dependent timevarying delay. $\left\{r_{t}, t \geqslant 0\right\}$ and $\left\{s_{t}, t \geqslant 0\right\}$ are continuous-time Markovian processes with right continuous trajectories and taking values in finite sets $S_{1}=\{1,2, \ldots, N\}, S_{2}=\{1,2, \ldots, M\}$ with the transition probability matrices $\Pi=\left[\pi_{i l}\right],\left(i, l \in S_{1}\right)$ and $\Lambda=\left[\lambda_{j k}\right],\left(j, k \in S_{2}\right)$, respectively, given by

$$
\begin{gathered}
\operatorname{Pr}\left\{r_{t+\Delta}=l \mid r_{t}=i\right\}= \begin{cases}\pi_{i l} \Delta+o(\Delta), & l \neq i, \\
1+\pi_{i i} \Delta+o(\Delta), & l=i,\end{cases} \\
\operatorname{Pr}\left\{s_{t+\Delta}=k \mid s_{t}=j\right\}= \begin{cases}\lambda_{j k} \Delta+o(\Delta), & k \neq j, \\
1+\lambda_{j j} \Delta+o(\Delta), & k=j,\end{cases}
\end{gathered}
$$

where $\Delta>0$ and $\lim _{\Delta \rightarrow 0}(o(\Delta) / \Delta)=0 ; \pi_{i l} \geqslant 0$ for $i \neq l$ is the transition rate from mode $i$ at time $t$ to mode $l$ at time $t+\Delta$ and $\pi_{i i}=-\sum_{l=1, l \neq i}^{N} \pi_{i l} ; \lambda_{j k} \geqslant 0$ for $j \neq k$ is the transition rate 
from mode $j$ at time $t$ to mode $k$ at time $t+\Delta$ and $\lambda_{j j}=-\sum_{k=1, k \neq j}^{M} \lambda_{j k}$. The processes $r_{t}$ and $s_{t}$ are assumed to be independent throughout this paper. For simplicity, a matrix $R\left(r_{t}\right)$ will be denoted by $R_{i}$. For example, $A\left(r_{t}\right)$ is denoted by $A_{i}, A_{1}\left(r_{t}\right)$ is denoted by $A_{1 i},\left(i \in S_{1}\right), \tau\left(s_{t}, t\right)$ is denoted by $\tau_{j}(t),\left(j \in S_{2}\right)$, and so on. When the mode is in $s_{t}=j$, the mode-dependent time-varying delay satisfies

$$
0<\tau_{j}(t) \leqslant \tau_{j} \leqslant \tau, \quad \dot{\tau}_{j}(t) \leqslant \mu_{j},
$$

where $\tau=\max \left\{\tau_{j}\right\}$.

Remark 2.1. In this work, we have assumed that the delay mode is different from the system mode. This is more powerful and desirable in modeling of real systems, because the reason for jump in delay value may not be the same as that for jump in other system parameters.

Remark 2.2. The generalized stochastic system (2.1) is quite general since it considers noise perturbations, discrete, and distributed time-varying delays and Markovian jump processes with different modes. To the best of our knowledge, the generalized stochastic system (2.1) has never been considered in the previous literature.

Remark 2.3. A fault detection system consists of a residual generator and an evaluation stage, including an evaluation function and a threshold. Therefore, the fault detection problem to be addressed in this paper can be stated as the following two steps. The first step is to design a suitable filter to reduce the effect of disturbances on residual signals and to enhance the influence of faults. The second step is to determine the residual evaluation function and an appropriate threshold.

In this study, the following full-order fault detection filter is considered:

$$
\begin{gathered}
\mathrm{d} x_{f}(t)=A_{f_{i j}} x_{f}(t) \mathrm{d} t+B_{f_{i j}} \mathrm{~d} y(t), \\
r(t)=C_{f_{i j}} x_{f}(t), \\
x_{f}(0)=0, \quad i \in S_{1}, j \in S_{2},
\end{gathered}
$$

where $x_{f}(t)$ is the filter state vector. $r(t)$ is its output which is sensitive to faults. $\left(A_{f_{i j}} B_{f_{i j}} C_{f_{i j}}\right)$ are appropriately dimensioned filter matrices to be determined.

To improve the sensitiveness of residual to fault, we add a weighting matrix function into the fault $f(t)$, that is, $F_{\omega}(s)=W(s) F(s)$, where $F(s)$ and $F_{\omega}(s)$ denote, respectively, the Laplace transforms of $f(t)$ and $f_{\omega}(t)$. One state-space realization of $F_{\omega}(s)=W(s) F(s)$ can be

$$
\begin{gathered}
\dot{x}_{\omega}(t)=A_{\omega} x_{\omega}(t)+B_{\omega} f(t), \\
f_{\omega}(t)=C_{\omega} x_{\omega}(t), \\
x_{\omega}(0)=0 .
\end{gathered}
$$


Denoting $r_{e}(t)=r(t)-f_{\omega}(t)$, then the overall dynamic system can be governed by the following augmented system:

$$
\begin{gathered}
\mathrm{d} \xi(t)=\left[\bar{A}_{i j} \xi(t)+\bar{A}_{1 i j} K \xi\left(t-\tau_{j}(t)\right)+\bar{A}_{2 i j} K \int_{t-\tau_{j}(t)}^{t} \xi(s) \mathrm{d} s+\bar{B}_{i j} w(t)\right] \mathrm{d} t \\
+\bar{G}_{i j} K \xi(t) \mathrm{d} \omega(t), r_{e}(t)=\bar{C}_{i j} \xi(t), \xi(t)=\tilde{\phi}(t), \quad \forall t \in[-\tau, 0],
\end{gathered}
$$

where $\xi(t)=\left[\begin{array}{lll}x^{T}(t) & x_{f}^{T}(t) & x_{\omega}(t)\end{array}\right]^{T}, w(t)=\left[\begin{array}{llll}u^{T}(t) & v^{T}(t) & f^{T}(t)\end{array}\right]^{T}, \tilde{\phi}(t)=\left[\begin{array}{lll}\phi^{T}(t) & 0^{T} & 0^{T}\end{array}\right]^{T}$, and

$$
\begin{array}{cc}
\bar{A}_{i j}=\left[\begin{array}{ccc}
A_{i} & 0 & 0 \\
B_{f_{i j}} C_{i} & A_{f_{i j}} & 0 \\
0 & 0 & A_{\omega}
\end{array}\right], & \bar{A}_{1 i j}=\left[\begin{array}{c}
A_{1 i} \\
B_{f_{i j}} C_{1 i} \\
0
\end{array}\right], \quad \bar{A}_{2 i j}=\left[\begin{array}{c}
A_{2 i} \\
B_{f_{i j}} C_{2 i} \\
0
\end{array}\right], \\
\bar{B}_{i j}=\left[\begin{array}{ccc}
B_{0 i} & B_{1 i} & B_{2 i} \\
0 & B_{f_{i j}} D_{1 i} & B_{f_{i j}} D_{2 i} \\
0 & 0 & B_{\omega}
\end{array}\right], & \bar{G}_{i j}=\left[\begin{array}{c}
G_{1 i} \\
B_{f_{i j}} G_{2 i} \\
0
\end{array}\right], \\
K=\left[\begin{array}{lll}
I & 0 & 0
\end{array}\right] .
\end{array}
$$

For simplicity, let

$$
\begin{gathered}
\varphi(t)=\bar{A}_{i j} \xi(t)+\bar{A}_{1 i j} K \xi\left(t-\tau_{j}(t)\right)+\bar{A}_{2 i j} K \int_{t-\tau_{j}(t)}^{t} \xi(s) \mathrm{d} s+\bar{B}_{i j} w(t), \\
g(t)=\bar{G}_{i j} K \xi(t) .
\end{gathered}
$$

Now, the problem of fault detection is transformed into an $H_{\infty}$ filtering problem for system (2.1), which is described as follows: given a prescribed level of disturbance attenuation $\gamma$, determine a series of filter matrices $A_{f_{i j}}, B_{f_{i j}}$, and $C_{f_{i j}}\left(i \in S_{1}, j \in S_{2}\right)$ such that the augmented system (2.6) is stochastically stable.

After designing a fault detection filter, the remaining important task is to evaluate the generated residual. One of widely adopted methods is to choose a residual evaluation function and a threshold. In this paper, residual evaluation function $f(r)$ and a threshold $J$ th are selected as

$$
f(r)=\int_{t_{0}}^{t_{0}+T} r^{T}(t) r(t) \mathrm{d} t, \quad J \mathrm{th}=\sup _{v(t) \in L_{2}, f(t)=0} E\left\{\int_{t_{0}}^{t_{0}+T} r^{T}(t) r(t) \mathrm{d} t\right\},
$$

where $\left[t_{0}, t_{0}+T\right]$ is the finite-time window, $T$ denotes the limited length, and $t_{0}$ denotes the initial evaluation time. The occurrence of fault can be detected by comparing $f(r)$ and $J$ th, according to the following logic:

$$
\begin{gathered}
f(r)>J \text { th } \Longrightarrow \text { Faults } \Longrightarrow \text { Alarm, } \\
f(r)<J \text { th } \Longrightarrow \text { No Faults. }
\end{gathered}
$$


The following lemma and definitions are introduced, which will be used in the proof of the main results.

Lemma 2.4 (see [42]). For any matrix $M>0$, scalar $\gamma>0$, vector function $\omega:[0, \gamma] \rightarrow R^{n}$ such that the integrations concerned are well defined, the following inequality holds:

$$
\left[\int_{0}^{\gamma} \omega^{T}(s) \mathrm{d} s\right] M\left[\int_{0}^{\gamma} \omega(s) \mathrm{d} s\right] \leqslant \gamma \int_{0}^{\gamma} \omega^{T}(s) M \omega(s) \mathrm{d} s .
$$

Definition 2.5. The filtering error system (2.6) with $w(t)=0$ is said to be stochastically stable, if, for every system mode $r_{t}$, every time-delay mode $s_{t}$ and all finite initial state $\widetilde{\phi}(t)$, the following relation holds: $\lim _{t \rightarrow \infty} E\left\{|\xi(t)|^{2}\right\}=0$.

Definition 2.6. Given a scalar $\gamma>0$, the filtering error system (2.6) is said to be stochastically stable with an $H_{\infty}$ performance $\gamma$, if, for every system mode $r_{t}$ and every time-delay mode $s_{t}$, the filtering error system (2.6) with $w(t)=0$ is stochastically stable, and, under zero initial condition, it satisfies $\left\|r_{e}\right\|_{2} \leqslant \gamma\|w\|_{2}$ for any nonzero $w(t) \in L_{2}[0, \infty]$.

It should be pointed out that the joint process $\left(\xi(t), r_{t}, s_{t}\right)$ is not Markovian. In order to cast our model into the frame work for a Markovion system, let us define a new Markovion process: $\xi_{t}(s)=\xi(t+s),-\tau \leqslant s \leqslant 0$, and then $\left\{\left(\xi_{t}, r_{t}, s_{t}\right), t \geqslant 0\right\}$ is Markovian process with the initial state $\left(\tilde{\phi}(\cdot), r_{0}, s_{0}\right)$.

Let $\mathbf{C}\left(\mathbb{R}^{n} \times \mathbb{R}^{n} \times \mathbb{R}^{+} \times S_{1} \times S_{2}\right)$ denote the family of all nonnegative functions $V(\xi, \xi t, t, i, j)$ on $\mathbb{R}^{n} \times \mathbb{R}^{n} \times \mathbb{R}^{+} \times S_{1} \times S_{2}$, which are continuously twice differentiable in $\xi$ and differentiable in $t$. If $V \in \mathbf{C}\left(\mathbb{R}^{n} \times \mathbb{R}^{n} \times \mathbb{R}^{+} \times S_{1} \times S_{2}\right)$, then, along the trajectory of system (2.6), we define an operator $\mathcal{L}(\cdot)$ from $\mathbb{R}^{n} \times \mathbb{R}^{n} \times \mathbb{R}^{+} \times S_{1} \times S_{2}$ to $\mathbb{R}$ by

$$
\begin{aligned}
\perp V\left(\xi, \xi_{t}, t, i, j\right)= & V_{t}\left(\xi, \xi_{t}, t, i, j\right)+V_{\xi}\left(\xi, \xi_{t}, t, i, j\right) \varphi(t)+\sum_{l \in S_{1}} \pi_{i l} V\left(\xi, \xi_{t}, t, l, j\right) \\
& +\sum_{k \in S_{2}} \lambda_{j k} V\left(\xi, \xi_{t}, t, i, k\right)+\frac{1}{2} \operatorname{trace}\left[g^{T}(t) V_{\xi \xi}\left(\xi, \xi_{t}, t, i, j\right) g(t)\right],
\end{aligned}
$$

where

$$
\begin{gathered}
V_{t}\left(\xi, \xi_{t}, t, i, j\right)=\frac{\partial V\left(\xi, \xi_{t}, t, i, j\right)}{\partial t}, \\
V_{\xi}\left(\xi, \xi_{t}, t, i, j\right)=\left(\frac{\partial V\left(\xi, \xi_{t}, t, i, j\right)}{\partial \xi_{1}}, \ldots, \frac{\partial V\left(\xi, \xi_{t}, t, i, j\right)}{\partial \xi_{n}}\right), \\
V_{\xi \xi}\left(\xi, \xi_{t}, t, i, j\right)=\left(\frac{\partial^{2} V\left(\xi, \xi_{t}, t, i, j\right)}{\partial \xi_{i} \xi_{j}}\right) .
\end{gathered}
$$




\section{Main Results}

In this section, we first propose a delay-dependent sufficient condition for stochastic stability with the $H_{\infty}$ performance of filtering error system (2.6). Now, define a stochastic Lyapunov functional candidate for systems (2.6) as

$$
V\left(\xi, \xi_{t}, t, i, j\right)=\sum_{n=1}^{6} V_{n}\left(\xi, \xi_{t}, t, i, j\right)
$$

where

$$
\begin{aligned}
V_{1}\left(\xi, \xi_{t}, t, i, j\right)= & \xi^{T}(t) P\left(r_{t}, s_{t}\right) \xi(t), \\
V_{2}\left(\xi, \xi_{t}, t, i, j\right)= & \int_{t-\tau\left(t, s_{t}\right)}^{t} \xi^{T}(s) K^{T} Q_{1}\left(r_{t}, s_{t}\right) K \xi(s) \mathrm{d} s \\
& +\int_{t-\tau\left(s_{t}\right)}^{t} \xi^{T}(s) K^{T} Q_{2}\left(r_{t}, s_{t}\right) K \xi(s) \mathrm{d} s, \\
V_{3}\left(\xi, \xi_{t}, t, i, j\right)= & \int_{t-\tau\left(t, s_{t}\right)}^{t}\left[\int_{\theta}^{t} \xi^{T}(s) K^{T} \mathrm{~d} s\right] R_{1}\left(r_{t}, s_{t}\right)\left[\int_{\theta}^{t} K \xi(s) \mathrm{d} s\right] \mathrm{d} \theta, \\
V_{4}\left(\xi, \xi_{t}, t, i, j\right)= & \int_{-\tau}^{0} \int_{t+\theta}^{t} \varphi^{T}(s) K^{T} Z K \varphi(s) \mathrm{d} s \mathrm{~d} \theta, \\
V_{5}\left(\xi, \xi_{t}, t, i, j\right)= & \int_{-\tau}^{0} \int_{t+\theta}^{t} \xi^{T}(s) K^{T} R_{2} K \xi(s) \mathrm{d} s \mathrm{~d} \theta, \\
V_{6}\left(\xi, \xi_{t}, t, i, j\right)= & \int_{0}^{\tau} \int_{-\theta}^{0} \int_{t+s}^{t} \xi^{T}(\alpha) K^{T} R_{3} K \xi(\alpha) \mathrm{d} \alpha \mathrm{d} s \mathrm{~d} \theta .
\end{aligned}
$$

By Itô's formula, we obtain the stochastic differential as

$$
\mathrm{d} V=\perp \sum_{n=1}^{6} V_{n}\left(\xi, \xi_{t}, t, i, j\right) \mathrm{d} t+2 \xi^{T}(t) P_{i j} g(t) \mathrm{d} \omega(t)
$$

where $\mathcal{L}$ is the weak infinitesimal generator of the random process $\left\{\xi_{t}, r_{t}, s_{t}\right\}$ along the system (2.6).

Using the operator (2.12), we have

$$
\begin{aligned}
\mathcal{L} V_{1}\left(\xi, \xi_{t}, t, i, j\right)= & 2 \xi^{T}(t) P_{i j}\left[\bar{A}_{i j} \xi(t)+\bar{A}_{1 i j} K \xi\left(t-\tau_{j}(t)\right)+\bar{A}_{2 i j} K \int_{t-\tau_{j}(t)}^{t} \xi(s) \mathrm{d} s+\bar{B}_{i j} w(t)\right] \\
& +\xi^{T}(t)\left[\sum_{l \in S_{1}} \pi_{i l} P_{l j}+\sum_{k \in S_{2}} \lambda_{j k} P_{i k}\right] \xi(t)+g^{T}(t) P_{i j} g(t) .
\end{aligned}
$$


The derivative of the first term in $V_{2}\left(\xi, \xi_{t}, t, i, j\right)$ is given as follows:

$$
\begin{aligned}
& \mathcal{L} \int_{t-\tau\left(t, s_{t}\right)}^{t} \xi^{T}(s) K^{T} Q_{1}\left(r_{t}, s_{t}\right) K \xi(s) \mathrm{d} s \\
&=\lim _{\Delta \rightarrow 0^{+}} \frac{1}{\Delta} E\left\{\int_{t+\Delta-\tau\left(s_{t+\Delta}, t+\Delta\right)}^{t+\Delta} \xi^{T}(s) K^{T} Q_{1}\left(r_{t+\Delta}, s_{t+\Delta}\right) K \xi(s) \mathrm{d} s\right. \\
&\left.\quad-\int_{t-\tau_{j}(t)}^{t} \xi^{T}(s) K^{T} Q_{1 i j} K \xi(s) \mathrm{d} s\right\} \\
&=\int_{t-\tau_{j}(t)}^{t} \xi^{T}(s) K^{T}\left(\sum_{l \in S_{1}} \pi_{i l} Q_{1 l j}\right) K \xi(s) \mathrm{d} s \\
& \quad+\sum_{k \in S_{2}} \lambda_{j k} \int_{t-\tau_{k}(t)}^{t} \xi^{T}(s) K^{T} Q_{1 i k} K \xi(s) \mathrm{d} s \\
& \quad+\xi^{T}(t) K^{T} Q_{1 i j} K \xi(t)-\left(1-\dot{\tau}_{j}(t)\right) \xi^{T}\left(t-\tau_{j}(t)\right) K^{T} Q_{1 i j} K \xi\left(t-\tau_{j}(t)\right) \\
& \leqslant \xi^{T}(t) K^{T} Q_{1 i j} K \xi(t)-\left(1-\mu_{j}\right) \xi^{T}\left(t-\tau_{j}(t)\right) K^{T} Q_{1 i j} K \xi\left(t-\tau_{j}(t)\right) \\
&+\int_{t-\tau_{j}(t)}^{t} \xi^{T}(s) K^{T}\left(\sum_{l \in S_{1}} \pi_{i l} Q_{1 l j}\right) K \xi(s) \mathrm{d} s \\
&+\lambda_{j j} \int_{t-\tau_{j}(t)}^{t} \xi^{T}(s) K^{T} Q_{1 i j} K \xi(s) \mathrm{d} s \\
&+\int_{t-\tau}^{t} \xi^{T}(s) K^{T}\left(\sum_{k \neq j} \lambda_{j k} Q_{1 i k}\right) K \xi(s) \mathrm{d} s .
\end{aligned}
$$

Following a similar method of (3.5), it is easy to obtain

$$
\begin{aligned}
& \mathcal{L} \int_{t-\tau\left(s_{t}\right)}^{t} \xi^{T}(s) K^{T} Q_{2}\left(r_{t}, s_{t}\right) K \xi(s) \mathrm{d} s \\
& =\xi^{T}(t) K^{T} Q_{2 i j} K \xi(t)-\xi^{T}\left(t-\tau_{j}\right) K^{T} Q_{2 i j} K \xi\left(t-\tau_{j}\right) \\
& \quad+\int_{t-\tau_{j}}^{t} \xi^{T}(s) K^{T}\left(\sum_{l \in S_{1}} \pi_{i l} Q_{2 l j}\right) K \xi(s) \mathrm{d} s \\
& \quad+\sum_{k \in S_{2}} \lambda_{j k} \int_{t-\tau_{k}}^{t} \xi^{T}(s) K^{T} Q_{2 i k} K \xi(s) \mathrm{d} s
\end{aligned}
$$


Journal of Applied Mathematics

9

$$
\begin{aligned}
\leqslant & \xi^{T}(t) K^{T} Q_{2 i j} K \xi(t)-\xi^{T}\left(t-\tau_{j}\right) K^{T} Q_{2 i j} K \xi\left(t-\tau_{j}\right) \\
& +\int_{t-\tau_{j}}^{t} \xi^{T}(s) K^{T}\left(\sum_{l \in S_{1}} \pi_{i l} Q_{2 l j}\right) K \xi(s) \mathrm{d} s \\
& +\lambda_{j j} \int_{t-\tau_{j}}^{t} \xi^{T}(s) K^{T} Q_{2 i j} K \xi(s) \mathrm{d} s \\
& +\int_{t-\tau}^{t} \xi^{T}(s) K^{T}\left(\sum_{k \neq j} \lambda_{j k} Q_{2 i k}\right) K \xi(s) \mathrm{d} s, \\
\perp V_{3}(\xi, & \left.\xi_{t}, t, i, j\right) \\
= & -\left(1-\dot{\tau}_{j}(t)\right) \int_{t-\tau_{j}(t)}^{t} \xi^{T}(s) K^{T} \mathrm{~d} s R_{1 i j} \int_{t-\tau_{j}(t)}^{t} K \xi(s) \mathrm{d} s+2 \int_{t-\tau_{j}(t)}^{t} \xi^{T}(t) K^{T} R_{1 i j} \\
& \times \int_{\theta}^{t} K \xi(s) \mathrm{d} s \mathrm{~d} \theta+\sum_{l \in S_{1}} \pi_{i l} \int_{t-\tau_{j}(t)}^{t}\left(\int_{\theta}^{t} \xi^{T}(s) K^{T} \mathrm{~d} s\right) R_{1 l j}\left(\int_{\theta}^{t} K \xi(s) \mathrm{d} s\right) \mathrm{d} \theta \\
& +\sum_{k \in S_{2}} \lambda_{j k} \int_{t-\tau_{k}(t)}^{t} \int_{\theta}^{t} \xi^{T}(s) K^{T} R_{1 i k} K \xi(s) \mathrm{d} s \mathrm{~d} \theta .
\end{aligned}
$$

Using Lemma 2.4 and considering (2.3), we have

$$
\begin{aligned}
\mathcal{L} V_{3}\left(\xi, \xi_{t}, t, i, j\right) & \\
\leqslant & -\left(1-\mu_{j}\right)\left(\int_{t-\tau_{j}(t)}^{t} \xi^{T}(s) K^{T} \mathrm{~d} s\right) R_{1 i j}\left(\int_{t-\tau_{j}(t)}^{t} K \xi(s) \mathrm{d} s\right)+\xi^{T}(t) K^{T}\left(\frac{1}{2} \tau_{j}^{2} R_{1 i j}\right) K \xi(t) \\
& +\int_{t-\tau_{j}(t)}^{t} \int_{\theta}^{t} \xi^{T}(s) K^{T} R_{1 i j} K \xi(s) \mathrm{d} s \mathrm{~d} \theta+\sum_{l \neq i} \pi_{i l} \int_{t-\tau_{j}(t)}^{t}(t-\theta) \int_{\theta}^{t} \xi^{T}(s) K^{T} R_{1 l j} K \xi(s) \mathrm{d} s \mathrm{~d} \theta \\
& +\sum_{k \neq j} \lambda_{j k} \int_{t-\tau_{k}(t)}^{t}(t-\theta) \int_{\theta}^{t} \xi^{T}(s) K^{T} R_{1 i k} K \xi(s) \mathrm{d} s \mathrm{~d} \theta \\
\leqslant & -\left(1-\mu_{j}\right)\left(\int_{t-\tau_{j}(t)}^{t} \xi^{T}(s) K^{T} \mathrm{~d} s\right) R_{1 i j}\left(\int_{t-\tau_{j}(t)}^{t} K \xi(s) \mathrm{d} s\right) \\
& +\xi^{T}(t) K^{T}\left(\frac{1}{2} \tau_{j}^{2} R_{1 i j}\right) K \xi(t) \\
& +\int_{t-\tau_{j}(t)}^{t} \int_{\theta}^{t} \xi^{T}(s) K^{T} R_{1 i j} K \xi(s) \mathrm{d} s \mathrm{~d} \theta+\sum_{l \neq i} \pi_{i l} \int_{t-\tau_{j}(t)}^{t} \xi^{T}(s) K^{T} R_{1 l j} K \xi(s) \\
& \times \int_{t-\tau_{j}(t)}^{t}(t-\theta) \mathrm{d} \theta \mathrm{d} s+\sum_{k \neq j}^{t} \lambda_{j k} \int_{t-\tau_{k}(t)}^{t} \xi^{T}(s) K^{T} R_{1 i k} K \xi(s) \int_{t-\tau_{k}(t)}^{t}(t-\theta) \mathrm{d} \theta \mathrm{d} s
\end{aligned}
$$




$$
\begin{aligned}
\leqslant & -\left(1-\mu_{j}\right)\left(\int_{t-\tau_{j}(t)}^{t} \xi^{T}(s) K^{T} \mathrm{~d} s\right) R_{1 i j}\left(\int_{t-\tau_{j}(t)}^{t} K \xi(s) \mathrm{d} s\right)+\xi^{T}(t) K^{T}\left(\frac{1}{2} \tau_{j}^{2} R_{1 i j}\right) K \xi(t) \\
& +\int_{t-\tau_{j}(t)}^{t} \int_{\theta}^{t} \xi^{T}(s) K^{T} R_{1 i j} K \xi(s) \mathrm{d} s \mathrm{~d} \theta+\frac{1}{2} \tau_{j}^{2} \sum_{l \neq i} \pi_{i l} \int_{t-\tau}^{t} \xi^{T}(s) K^{T} R_{1 l j} K \xi(s) \mathrm{d} s \\
& +\frac{1}{2} \sum_{k \neq j} \tau_{k}^{2} \lambda_{j k} \int_{t-\tau}^{t} \xi^{T}(s) K^{T} R_{1 i k} K \xi(s) \mathrm{d} s .
\end{aligned}
$$

Moreover,

$$
\begin{aligned}
& \mathcal{L} V_{4}\left(\xi, \xi_{t}, t, i, j\right)=\tau \varphi^{T}(t) K^{T} Z K \varphi(t)-\int_{t-\tau}^{t} \varphi^{T}(s) K^{T} Z K \varphi(s) \mathrm{d} s, \\
& \mathcal{L} V_{5}\left(\xi, \xi_{t}, t, i, j\right)=\tau \xi^{T}(t) K^{T} R_{2} K \xi(t)-\int_{t-\tau}^{t} \xi^{T}(s) K^{T} R_{2} K \xi(s) \mathrm{d} s, \\
& \mathcal{L} V_{6}\left(\xi, \xi_{t}, t, i, j\right)=\frac{1}{2} \tau^{2} \xi^{T}(t) K^{T} R_{3} K \xi(t)-\int_{t-\tau}^{t} \int_{\theta}^{t} \xi^{T}(s) K^{T} R_{3} K \xi(s) \mathrm{d} s \mathrm{~d} \theta .
\end{aligned}
$$

We define

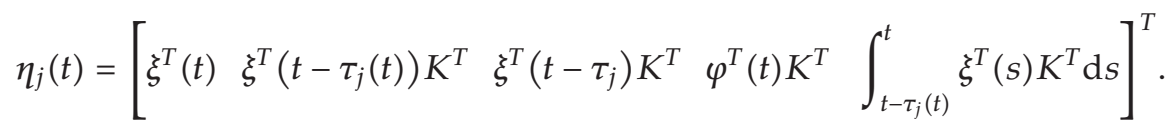

The following equations are true for any matrices $\mathbf{L}, \mathbf{M}, \mathbf{N}$, and $\mathbf{Y}$ with appropriate dimensions:

$$
\begin{aligned}
& 0=2 \eta_{j}^{T}(t) \mathbf{L}\left[K \xi(t)-K \xi\left(t-\tau_{j}(t)\right)-\int_{t-\tau_{j}(t)}^{t} K \tilde{\varphi}(s) \mathrm{d} s-\int_{t-\tau_{j}(t)}^{t} K \tilde{g}(s) \mathrm{d} \omega(s)\right], \\
& 0=2 \eta_{j}^{T}(t) \mathbf{M}\left[K \xi\left(t-\tau_{j}(t)\right)-K \xi\left(t-\tau_{j}\right)-\int_{t-\tau_{j}}^{t-\tau_{j}(t)} K \tilde{\varphi}(s) \mathrm{d} s-\int_{t-\tau_{j}}^{t-\tau_{j}(t)} K \tilde{g}(s) \mathrm{d} \omega(s)\right], \\
& 0=2 \eta_{j}^{T}(t) \mathbf{N}\left[-K \varphi(t)+K \bar{A}_{i j} \xi(t)+K \bar{A}_{1 i j} K \xi\left(t-\tau_{j}(t)\right)+K \bar{A}_{2 i j} K \int_{t-\tau_{j}(t)}^{t} \xi(s) \mathrm{d} s+K \bar{B}_{1 i j} v(t)\right], \\
& 0=\tau_{j} \eta_{j}^{T}(t) \mathbf{Y} \eta_{j}(t)-\int_{t-\tau_{j}}^{t-\tau_{j}(t)} \eta_{j}^{T}(t) \mathbf{Y} \eta_{j}(t) \mathrm{d} s-\int_{t-\tau_{j}(t)}^{t} \eta_{j}^{T}(t) \mathbf{Y} \eta_{j}(t) \mathrm{d} s,
\end{aligned}
$$


where

$$
\begin{gathered}
\mathbf{L}=\left[\begin{array}{lllll}
L_{1}^{T} K & L_{2}^{T} & L_{3}^{T} & L_{4}^{T} & L_{5}^{T}
\end{array}\right]^{T}, \quad \mathbf{M}=\left[\begin{array}{lllll}
M_{1}^{T} K & M_{2}^{T} & M_{3}^{T} & M_{4}^{T} & M_{5}^{T}
\end{array}\right]^{T}, \\
\mathbf{N}=\left[\begin{array}{lllll}
0 & 0 & 0 & N^{T} & 0
\end{array}\right]^{T}, \\
\mathbf{Y}=\left[\begin{array}{ccccc}
K^{T} Y_{11} K & K^{T} Y_{12} & K^{T} Y_{13} & K^{T} Y_{14} & K^{T} Y_{15} \\
* & Y_{22} & Y_{23} & Y_{24} & \Upsilon_{25} \\
* & * & Y_{33} & Y_{34} & \Upsilon_{35} \\
* & * & * & Y_{44} & \Upsilon_{45} \\
* & * & * & * & Y_{55}
\end{array}\right] .
\end{gathered}
$$

Considering (3.4)-(3.11), we obtain that

$$
\begin{aligned}
\mathcal{L} V(\xi, & \left.\xi_{t}, i, j\right) \\
\leqslant & {\left[\begin{array}{c}
\eta_{j}(t) \\
w(t)
\end{array}\right]^{T}\left[\begin{array}{cc}
\Sigma_{i j} & \Phi_{1 i j} \\
* & 0
\end{array}\right]\left[\begin{array}{l}
\eta_{j}(t) \\
w(t)
\end{array}\right] } \\
& +\int_{t-\tau_{j}(t)}^{t} \xi^{T}(s) K^{T}\left[\sum_{l \in S_{1}, l \neq i} \pi_{i l}\left(Q_{1 l j}+\frac{\tau_{j}^{2}}{2} R_{1 l j}\right)+\pi_{i i} Q_{1 i j}+\lambda_{j j} Q_{1 i j}\right] K \xi(s) \mathrm{d} s \\
& +\int_{t-\tau}^{t} \xi^{T}(s) K^{T}\left[\sum_{k \in S_{2}, k \neq j} \lambda_{j k}\left(Q_{1 i k}+Q_{2 i k}+\frac{\tau_{k}^{2}}{2} R_{1 i k}\right)-R_{2}\right] K \xi(s) \mathrm{d} s \\
& +\int_{t-\tau_{j}}^{t} \xi^{T}(s) K^{T}\left(\begin{array}{c}
\left.\sum_{l \in S_{1}} \pi_{i l} Q_{2 l j}+\lambda_{j j} Q_{2 i j}\right) K \xi(s) \mathrm{d} s \\
\end{array}\right. \\
& +\int_{t-\tau}^{t} \int_{\theta}^{t} \xi^{T}(s) K^{T}\left(\begin{array}{c}
\left.R_{1 i j}-R_{3}\right) K \xi(s) \mathrm{d} s \mathrm{~d} \theta \\
\end{array}\right. \\
& -\int_{t-\tau_{j}(t)}^{t}\left[\begin{array}{c}
\eta_{j}(t) \\
K \varphi(s)
\end{array}\right]^{T}\left[\begin{array}{cc}
\mathbf{Y} & \mathbf{L} \\
* & Z
\end{array}\right]\left[\begin{array}{c}
\eta_{j}(t) \\
K \varphi(s)
\end{array}\right] \mathrm{d} s \\
& -\int_{t-\tau_{j}}^{t-\tau_{j}(t)}\left[\begin{array}{c}
\eta_{j}(t) \\
K \varphi(s)
\end{array}\right]^{T}\left[\begin{array}{cc}
\mathbf{Y} & \mathbf{M} \\
* & Z
\end{array}\right]\left[\begin{array}{c}
\eta_{j}(t) \\
K \varphi(s)
\end{array}\right] \mathrm{d} s+\tilde{f}(t),
\end{aligned}
$$


where

$$
\begin{aligned}
& \tilde{f}(t)=2 \xi^{T}(t) P_{i j} g(t) \mathrm{d} \omega(t)-2 \eta_{j}^{T}(t) \mathbf{L} \int_{t-\tau_{j}(t)}^{t} K g(s) \mathrm{d} \omega(s)-2 \eta_{j}^{T}(t) \mathbf{M} \int_{t-\tau_{j}}^{t-\tau_{j}(t)} K g(s) \mathrm{d} \omega(s), \\
& \Sigma_{i j}=\left[\begin{array}{ccccc}
\Sigma_{11} & \Sigma_{12} & \Sigma_{13} & \Sigma_{14} & \Sigma_{15} \\
* & \Sigma_{22} & \Sigma_{23} & \Sigma_{24} & \Sigma_{25} \\
* & * & \Sigma_{33} & \Sigma_{34} & \Sigma_{35} \\
* & * & * & \Sigma_{44} & \Sigma_{45} \\
* & * & * & * & \Sigma_{55}
\end{array}\right], \\
& \Sigma_{11}=P_{i j} \bar{A}_{i j}+\bar{A}_{i j}^{T} P_{i j}+K^{T} \bar{G}_{i j}^{T} P_{i j} \bar{G}_{i j} K+\sum_{l \in S_{1}} \pi_{i l} P_{l j}+\sum_{k \in S_{2}} \lambda_{j k} P_{i k} \\
& +K^{T}\left(Q_{1 i j}+Q_{2 i j}+\tau R_{2}+\frac{1}{2} \tau^{2} R_{3}+\frac{1}{2} \tau_{j}^{2} R_{1 i j}+L_{1}+L_{1}^{T}+\tau_{j} Y_{11}\right) K, \\
& \Sigma_{12}=P_{i j} \bar{A}_{1 i j}+K^{T}\left(-L_{1}+L_{2}^{T}+M_{1}+\tau_{j} Y_{12}\right), \\
& \Sigma_{13}=K^{T}\left(L_{3}^{T}-M_{1}+\tau_{j} Y_{13}\right), \\
& \Sigma_{14}=K^{T}\left(L_{4}^{T}+\tau_{j} Y_{14}\right)+\bar{A}_{i j}^{T} K^{T} N^{T}, \\
& \Sigma_{15}=P_{i j} \bar{A}_{2 i j}+K^{T}\left(L_{5}^{T}+\tau_{j} Y_{15}\right), \\
& \Sigma_{22}=-\left(1-\mu_{j}\right) Q_{1 i j}-L_{2}-L_{2}^{T}+M_{2}+M_{2}^{T}+\tau_{j} Y_{22} \text {, } \\
& \Sigma_{23}=-L_{3}^{T}-M_{2}+M_{3}^{T}+\tau_{j} Y_{23}, \\
& \Sigma_{24}=-L_{4}^{T}+M_{4}^{T}+\bar{A}_{1 i j}^{T} K^{T} N^{T}+\tau_{j} Y_{24}, \\
& \Sigma_{25}=-L_{5}^{T}+M_{5}^{T}+\tau_{j} Y_{25} \text {, } \\
& \Sigma_{33}=-Q_{2 i j}-M_{3}-M_{3}^{T}+\tau_{j} Y_{33}, \\
& \Sigma_{34}=-M_{4}^{T}+\tau_{j} Y_{34}, \\
& \Sigma_{35}=-M_{5}^{T}+\tau_{j} Y_{35}, \\
& \Sigma_{44}=\tau Z-N-N^{T}+\tau_{j} Y_{44}, \\
& \Sigma_{45}=N K \bar{A}_{2 i j}+\tau_{j} Y_{45}, \\
& \Sigma_{55}=-\left(1-\mu_{j}\right) R_{1 i j}+\tau_{j} Y_{55},
\end{aligned}
$$

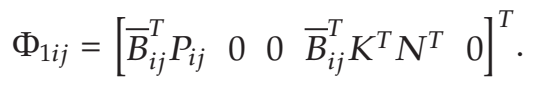

Therefore, we have the following result for the $H_{\infty}$ performance analysis. 
Theorem 3.1. Given scalars $\tau, \tau_{j}$, and $\mu_{j}$, the fault detection system (2.6) is stochastically stable with an $H_{\infty}$ performance $\gamma$ for any time delay $\tau_{j}(t)$ satisfying (2.3), if there exist matrices $P_{i j}>0, Z>0$, $Q_{1 i j}>0, Q_{2 i j}>0, R_{1 i j}>0, R_{2}>0, R_{3}>0$, and matrices $\mathbf{L}, \mathbf{M}, \mathbf{N}$, Y denoted in (3.10)-(3.11) such that for each $i \in S_{1}, j \in S_{2}$

$$
\begin{gathered}
{\left[\begin{array}{cccc}
\tilde{\Sigma}_{i j} & \Phi_{1 i j} & \Phi_{2 i j} & \Phi_{3 i j} \\
* & -\gamma^{2} I & 0 & 0 \\
* & * & -I & 0 \\
* & * & * & -P_{i j}
\end{array}\right]<0} \\
\sum_{l \in S_{1}, l \neq i} \pi_{i l}\left(Q_{1 l j}+\frac{\tau_{j}^{2}}{2} R_{1 l j}\right)+\pi_{i i} Q_{1 i j}+\lambda_{j j} Q_{1 i j}<0, \\
\sum_{k \in S_{2}, k \neq j} \lambda_{j k}\left(Q_{1 i k}+Q_{2 i k}+\frac{\tau_{k}^{2}}{2} R_{1 i k}\right)-R_{2}<0, \\
\sum_{l \in S_{1}} \pi_{i l} Q_{2 l j}+\lambda_{j j} Q_{2 i j}<0 \\
R_{1 i j}-R_{3}<0, \\
{\left[\begin{array}{ll}
\mathbf{Y} & \mathbf{L} \\
* & Z
\end{array}\right] \geqslant 0, \quad\left[\begin{array}{cc}
\mathbf{Y} & \mathbf{M} \\
* & Z
\end{array}\right] \geqslant 0,}
\end{gathered}
$$

where

$$
\begin{gathered}
\tilde{\Sigma}_{i j}=\left[\begin{array}{cccccc}
\Sigma_{11}-K^{T} \bar{G}_{i j}^{T} P_{i j} \bar{G}_{i j} K & \Sigma_{12} & \Sigma_{13} & \Sigma_{14} & \Sigma_{15} \\
* & \Sigma_{22} & \Sigma_{23} & \Sigma_{24} & \Sigma_{25} \\
* & * & \Sigma_{33} & \Sigma_{34} & \Sigma_{35} \\
* & * & * & \Sigma_{44} & \Sigma_{45} \\
* & * & * & * & \Sigma_{55}
\end{array}\right], \\
\Phi_{2 i j}=\left[\begin{array}{lllll}
\bar{C}_{i j} & 0 & 0 & 0 & 0
\end{array}\right]^{T}, \quad \Phi_{3 i j}=\left[\begin{array}{llllll}
P_{i j} \bar{G}_{i j} K & 0 & 0 & 0 & 0
\end{array}\right]^{T} .
\end{gathered}
$$

Proof. Using Schur complement formula to (3.15), it can be seen that (3.15) is equivalent to

$$
\left[\begin{array}{cc}
\Sigma_{i j}+\Phi_{2 i j} \Phi_{2 i j}^{T} & \Phi_{1 i j} \\
* & -\gamma^{2} I
\end{array}\right]<0
$$

Now, we show that the filtering error system (2.6) with $w(t)=0$ is stochastically stable. If $w(t)=0$, from (3.13) and (3.16)-(3.17), we can obtain

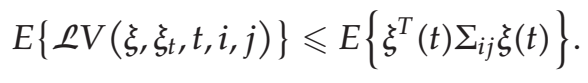


Inequality (3.20) implies that $\Sigma_{i j}<0$. Thus, we have

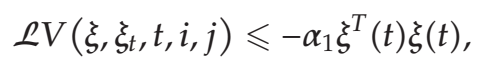

where $\alpha_{1}=\min _{i \in S_{1}, j \in S_{2}}\left\{\lambda_{\min }\left(-\Sigma_{i j}\right)\right\}>0$. Therefore, for any $T>0$, by Dynkin's formula, we have

$$
\int_{0}^{T} E\left\{\xi^{T}(s) \xi(s)\right\} \mathrm{d} s \leqslant \alpha_{1}^{-1} V\left(\tilde{\phi}(0), r_{0}, s_{0}\right)
$$

which means that $\lim _{t \rightarrow \infty} E\left\{|\xi(t)|^{2}\right\}=0$. Thus, the filtering error system (2.6) with $w(t)=0$ is stochastically stable by Definition 2.5 .

In the sequel, we will deal with the $H_{\infty}$ performance of the filtering error system (2.6). Using (3.19) and $H_{\infty}$ performance, we have

$$
E\left\{\mathcal{L}\left(\xi, \xi_{t}, t, i, j\right)+r_{e}^{T}(t) r_{e}(t)-\gamma^{2} w^{T}(t) w(t)\right\} \leqslant\left[\begin{array}{l}
\eta_{j}(t) \\
w(t)
\end{array}\right]^{T}\left[\begin{array}{cc}
\Sigma_{i j}+\Phi_{2 i j} \Phi_{2 i j}^{T} & \Phi_{1 i j} \\
* & -\gamma^{2} I
\end{array}\right]\left[\begin{array}{l}
\eta_{j}(t) \\
w(t)
\end{array}\right]<0
$$

Noting that the zero initial condition, then it follows from (3.23) that

$$
\begin{aligned}
& J_{H}=E\left\{\int_{0}^{\infty}\left[r_{e}^{T}(t) r_{e}(t)-r^{2} w^{T}(t) w(t)\right] \mathrm{d} t\right\} \\
& \leqslant E\left\{\int_{0}^{\infty}\left[r_{e}^{T}(t) r_{e}(t)-r^{2} w^{T}(t) w(t)+\mathcal{L} V\left(\xi, \xi_{t}, t, i, j\right)\right] \mathrm{d} t\right\} \\
& <0 \text {. }
\end{aligned}
$$

Hence, if (3.15)-(3.17) hold, $J_{H}<0$ can be guaranteed. That is, $\left\|r_{e}\right\|_{2} \leqslant \gamma\|w\|_{2}$ for all nonzero $w(t)$. Therefore, the filtering error system (2.6) is stochastically stable with the $H_{\infty}$ performance $\gamma$ by Definition 2.6. This completes the proof.

Remark 3.2. Theorem 3.1 presents a new stochastic stability criterion by employing a novel mixed mode-dependent Lyapunov functional. The Lyapunov functional in this paper uses all information about $r_{t}, s_{t}$, and $\tau\left(t, s_{t}\right)$. Also, the Lyapunov matrices $P\left(r_{t}, s_{t}\right), Q_{1}\left(r_{t}, s_{t}\right)$, $Q_{2}\left(r_{t}, s_{t}\right)$, and $R_{1}\left(r_{t}, s_{t}\right)$ depend on both the system mode $r_{t}$ and the delay mode $s_{t}$. Hence, the Lyapunov functional in this paper is more general, and the condition on stability is more applicable. In the most published papers about Markovian jump systems with mixed time delays, the authors choose the mode-independent Lyapunov matrices which may lead to some conservativeness, such as $[15,17,23,31,33,37-39]$, to name a few among many important results in the literature. But, the selected mode-dependent Lyapunov matrices in 
this paper can reduce some conservativeness because they allow more freedom in choosing feasible solutions of LMIs.

Remark 3.3. In Theorem 3.1, $\mu_{j}<1$ can be extended to a wider range $\mu_{j}<\infty$ by dealing with the integral term $\lambda_{j j} \int_{t-\tau_{j}(t)}^{t} \xi^{T}(s) K^{T} Q_{1 i j} K \xi(s) \mathrm{d} s$ in (3.5). Noting that $\lambda_{j j}<0$, utilizing Lemma 2.4, one has

$$
\lambda_{j j} \int_{t-\tau_{j}(t)}^{t} \xi^{T}(s) K^{T} Q_{1 i j} K \xi(s) \mathrm{d} s \leqslant \frac{\lambda_{j j}}{\tau_{j}} \int_{t-\tau_{j}}^{t} \xi^{T}(s) K^{T} \mathrm{~d} s Q_{1 i j} \int_{t-\tau_{j}(t)}^{t} K \xi(s) \mathrm{d} s .
$$

Further, deleting $\lambda_{j j} Q_{1 i j}$ in (3.16) and adding $\left(\lambda_{j j} / \tau_{j}\right) Q_{1 i j}$ to $\Sigma_{55}$ in (3.15), we can obtain a more general stability condition.

Based on Theorem 3.1, the fault detection filter synthesis problem can be developed in terms of LMIs for the system (2.1) with different system and delay modes.

Theorem 3.4. Consider the system (2.1). Given scalars $\tau, \tau_{j}$, and $\mu_{j}$, the fault detection system (2.6) is stochastically stable with an $H_{\infty}$ performance $\gamma$ for any time delay $\tau_{j}(t)$ satisfying $(2.3)$ if there exist matrices $V_{i j}>0, W_{i j}>0, U_{i j}>0, Z>0, Q_{1 i j}>0, Q_{2 i j}>0, R_{1 i j}>0, R_{2}>0, R_{3}>0, \bar{A}_{f i j}$, $\bar{B}_{f i j}, \bar{C}_{f i j}$ and matrices $\mathbf{L}, \mathbf{M}, \mathbf{N}, \mathbf{Y}$ denoted in (3.10)-(3.11) such that for each $i \in S_{1}, j \in S_{2}$

$$
\begin{gathered}
{\left[\begin{array}{cccc}
\bar{\Sigma}_{i j} & \bar{\Phi}_{1 i j} & \bar{\Phi}_{2 i j} & \bar{\Phi}_{3 i j} \\
* & -\gamma^{2} I & 0 & 0 \\
* & * & -I & 0 \\
* & * & * & -\bar{\Phi}_{4 i j}
\end{array}\right]<0} \\
\sum_{l \in S_{1}, l \neq i} \pi_{i l}\left(Q_{1 l j}+\frac{\tau_{j}^{2}}{2} R_{1 l j}\right)+\pi_{i i} Q_{1 i j}+\lambda_{j j} Q_{1 i j}<0, \\
\sum_{k \in S_{2}, k \neq j} \lambda_{j k}\left(Q_{1 i k}+Q_{2 i k}+\frac{\tau_{k}^{2}}{2} R_{1 i k}\right)-R_{2}<0, \\
\sum_{l \in S_{1}} \pi_{i l} Q_{2 l j}+\lambda_{j j} Q_{2 i j}<0 \\
R_{1 i j}-R_{3}<0, \\
{\left[\begin{array}{ll}
\mathbf{Y} & \mathbf{L} \\
* & Z
\end{array}\right] \geqslant 0, \quad\left[\begin{array}{ll}
\mathbf{Y} & \mathbf{M} \\
* & Z
\end{array}\right] \geqslant 0}
\end{gathered}
$$


where

$$
\begin{aligned}
& \bar{\Sigma}_{i j}=\left[\begin{array}{ccccccc}
\bar{\Sigma}_{11} & \bar{\Sigma}_{12} & 0 & \bar{\Sigma}_{14} & \bar{\Sigma}_{15} & \bar{\Sigma}_{16} & \bar{\Sigma}_{17} \\
* & \bar{\Sigma}_{22} & 0 & \bar{\Sigma}_{24} & 0 & 0 & \bar{\Sigma}_{27} \\
* & * & \bar{\Sigma}_{33} & 0 & 0 & 0 & 0 \\
* & * & * & \bar{\Sigma}_{44} & \bar{\Sigma}_{45} & \bar{\Sigma}_{46} & \bar{\Sigma}_{47} \\
* & * & * & * & \bar{\Sigma}_{55} & \bar{\Sigma}_{56} & \bar{\Sigma}_{57} \\
* & * & * & * & * & \bar{\Sigma}_{66} & \bar{\Sigma}_{67} \\
* & * & * & * & * & * & \bar{\Sigma}_{77}
\end{array}\right], \quad \bar{\Phi}_{1 i j}=\left[\begin{array}{ccc}
V_{i j} B_{0 i} & V_{i j} B_{1 i} & V_{i j} B_{2 i} \\
0 & \bar{B}_{f i j} D_{1 i} & \bar{B}_{f i j} D_{2 i} \\
0 & 0 & U_{i j} B_{\omega} \\
0 & 0 & 0 \\
0 & 0 & 0 \\
N B_{0 i} & N B_{0 i} & N B_{0 i} \\
0 & 0 & 0
\end{array}\right], \\
& \bar{\Phi}_{2 i j}=\left[\begin{array}{c}
0 \\
\bar{C}_{f i j}^{T} \\
-C_{\omega}^{T} \\
0 \\
0 \\
0 \\
0
\end{array}\right], \quad \bar{\Phi}_{3 i j}=\left[\begin{array}{ccc}
G_{1 i}^{T} V_{i j}^{T} & G_{2 i}^{T} \bar{B}_{f i j}^{T} & 0 \\
0 & 0 & 0 \\
0 & 0 & 0 \\
0 & 0 & 0 \\
0 & 0 & 0 \\
0 & 0 & 0 \\
0 & 0 & 0
\end{array}\right], \quad \bar{\Phi}_{4 i j}=\left[\begin{array}{ccc}
V_{i j} & 0 & 0 \\
* & W_{i j} & 0 \\
* & * & U_{i j}
\end{array}\right] \text {, } \\
& \bar{\Sigma}_{11}=V_{i j} A_{i}+A_{i}^{T} V_{i j}+\sum_{l \in S_{1}} \pi_{i l} V_{l j}+\sum_{k \in S_{2}} \lambda_{j k} V_{i k}+Q_{1 i j}+Q_{2 i j}+\tau R_{2} \\
& +\frac{1}{2} \tau^{2} R_{3}+\frac{1}{2} \tau_{j}^{2} R_{1 i j}+L_{1}+L_{1}^{T}+\tau_{j} Y_{11} \\
& \bar{\Sigma}_{12}=C_{i}^{T} \bar{B}_{f_{i j}}^{T}, \quad \bar{\Sigma}_{14}=V_{i j} A_{1 i}-L_{1}+M_{1}+L_{2}^{T}+\tau_{j} Y_{12}, \\
& \bar{\Sigma}_{15}=L_{3}^{T}-M_{1}+\tau_{j} Y_{13}, \quad \bar{\Sigma}_{16}=L_{4}^{T}+\tau_{j} Y_{14}+A_{i}^{T} N^{T}, \\
& \bar{\Sigma}_{17}=V_{i j} A_{2 i}+L_{5}^{T}+\tau_{j} Y_{15}, \quad \bar{\Sigma}_{22}=\bar{A}_{f_{i j}}+\bar{A}_{f_{i j}}^{T}+\sum_{l \in S_{1}} \pi_{i l} W_{l j}+\sum_{k \in S_{2}} \lambda_{j k} W_{i k}, \\
& \bar{\Sigma}_{24}=\bar{B}_{f_{i j}} C_{1 i}, \quad \bar{\Sigma}_{27}=\bar{B}_{f_{i j}} C_{2 i}, \quad \bar{\Sigma}_{33}=U_{i j} A_{\omega}+A_{\omega}^{T} U_{i j}+\sum_{l \in S_{1}} \pi_{i l} U_{l j}+\sum_{k \in S_{2}} \lambda_{j k} U_{i k}, \\
& \bar{\Sigma}_{44}=-\left(1-\mu_{j}\right) Q_{1 i j}-L_{2}-L_{2}^{T}+M_{2}+M_{2}^{T}+\tau_{j} Y_{22} \text {, } \\
& \bar{\Sigma}_{45}=-L_{3}^{T}-M_{2}+M_{3}^{T}+\tau_{j} Y_{23} \text {, } \\
& \bar{\Sigma}_{46}=-L_{4}^{T}+M_{4}^{T}+\tau_{j} Y_{24}+A_{1 i}^{T} N^{T}, \quad \bar{\Sigma}_{47}=-L_{5}^{T}+M_{5}^{T}+\tau_{j} Y_{25}, \\
& \bar{\Sigma}_{55}=-Q_{2 i j}-M_{3}-M_{3}^{T}+\tau_{j} Y_{33}, \quad \bar{\Sigma}_{56}=-M_{4}^{T}+\tau_{j} Y_{34}, \quad \bar{\Sigma}_{57}=-M_{5}^{T}+\tau_{j} Y_{35}, \\
& \bar{\Sigma}_{66}=\tau Z-N^{T}-N+\tau_{j} Y_{44}, \quad \bar{\Sigma}_{67}=N A_{2 i}+\tau_{j} Y_{45}, \quad \bar{\Sigma}_{77}=-\left(1-\mu_{j}\right) R_{1 i j}+\tau_{j} Y_{55} .
\end{aligned}
$$

In this case, the parameters of the desired fault detection filter can be chosen by

$$
A_{f_{i j}}=W_{i j}^{-1} \bar{A}_{f_{i j}}, \quad B_{f_{i j}}=W_{i j}^{-1} \bar{B}_{f_{i j}}, \quad C_{f_{i j}}=\bar{C}_{f_{i j}} .
$$


Proof. For each $r_{t}=i \in S_{1}, s_{t}=j \in S_{2}$, we define a matrix $P_{i j}>0$ by $P_{i j}=\operatorname{diag}\left[\begin{array}{lll}V_{i j} & W_{i j} & U_{i j}\end{array}\right]$. Then, with the parameters in (3.28), it can be verified that, for each $i \in S_{1}, j \in S_{2}$, the LMI (3.26) can be rewritten as (3.15). Then, we can obtain the results in Theorem 3.1. This completes the proof.

Remark 3.5. Noting that the first diagonal element $\Sigma_{11}$ in (3.15) includes $P_{i j}, P_{l j}$, and $P_{i k}$. Owing to the restrictions on the authors' knowledge and the technique difficulties, $P_{i j}$ is assumed to be diagonal matrices to obtain the parameters of the fault detection filter. Although this assumption may cause some conservativeness, considering complete $P_{i j}$ results in bilinear matrix inequalities and not LMIs which are more conservative.

\section{A Numerical Example}

In this section, a numerical example will be presented to show the validity of the main results derived above.

Example 4.1. Let us consider the stochastic system (2.1) with the following system of matrices:

$$
\begin{aligned}
& A(1)=\left[\begin{array}{cc}
-10 & 0 \\
0.6 & -12
\end{array}\right], \quad A_{1}(1)=\left[\begin{array}{cc}
-1 & 0.3 \\
2 & -1
\end{array}\right], \quad A_{2}(1)=0.5 I, \quad B_{0}(1)=\left[\begin{array}{l}
0.1 \\
0.2
\end{array}\right], \\
& B_{1}(1)=\left[\begin{array}{l}
0.7 \\
0.1
\end{array}\right], \quad B_{2}(1)=\left[\begin{array}{c}
0.6 \\
0
\end{array}\right], \quad G_{1}(1)=I, \quad C(1)=\left[\begin{array}{ll}
2 & 2.1
\end{array}\right], \\
& C_{1}(1)=\left[\begin{array}{ll}
1.5 & 0
\end{array}\right], \quad C_{2}(1)=\left[\begin{array}{ll}
0.1 & 0.1
\end{array}\right], \quad D_{1}(1)=0.1, \quad D_{2}(1)=0.2, \\
& G_{2}(1)=\left[\begin{array}{ll}
-0.5 & -0.5
\end{array}\right], \\
& A(2)=\left[\begin{array}{cc}
-12 & 1 \\
-2 & -14.3
\end{array}\right], \quad A_{1}(2)=\left[\begin{array}{cc}
-1 & 1.3 \\
0.7 & -1.1
\end{array}\right], \quad A_{2}(2)=0.1 I, \quad B_{0}(2)=\left[\begin{array}{l}
0.1 \\
0.2
\end{array}\right], \\
& B_{1}(2)=\left[\begin{array}{ll}
0.1 \\
0.3
\end{array}\right], \quad B_{2}(2)=\left[\begin{array}{c}
0.3 \\
0
\end{array}\right], \quad G_{1}(2)=\left[\begin{array}{ll}
1 & 0 \\
1 & 1
\end{array}\right], \quad C(2)=\left[\begin{array}{ll}
2 & 2
\end{array}\right], \\
& C_{1}(2)=\left[\begin{array}{ll}
1.2 & 0.7
\end{array}\right], \quad C_{2}(2)=\left[\begin{array}{ll}
0.1 & 0.1
\end{array}\right], \quad D_{1}(2)=0.2, \quad D_{2}(2)=0.2, \\
& G_{2}(2)=\left[\begin{array}{ll}
-0.5 & -0.5
\end{array}\right] .
\end{aligned}
$$

The transition probability matrix is considered as

$$
\Pi=\left[\begin{array}{cc}
-5 & 5 \\
3 & -3
\end{array}\right], \quad \Lambda=\left[\begin{array}{cc}
-0.6 & 0.6 \\
0.5 & -0.5
\end{array}\right]
$$

In this example, the weighting matrix $W(s)$ in $F_{\omega}(s)=W(s) F(s)$ is supposed to be $W(s)=$ $5 /(s+5)$. Its state-space realization is given as (2.5) with $A_{\omega}=-5, B_{\omega}=5$ and $C_{\omega}=1$. Also, 


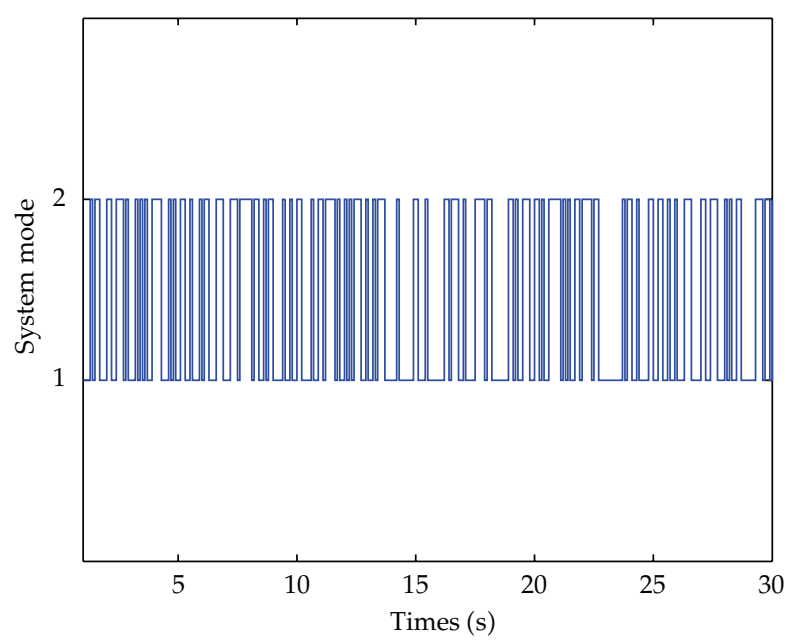

Figure 1: System jumping mode.

we assume that $\tau_{1}=0.6, \tau_{2}=0.4, \mu_{1}=0.4, \mu_{2}=0.3$. For $\gamma=2.0$, by the Theorem 3.4 in this paper, the filter matrices are obtained as

$$
\begin{aligned}
& A_{f_{11}}=\left[\begin{array}{cc}
-3.1724 & 1.1747 \\
-1.1416 & -0.5011
\end{array}\right], \quad B_{f_{11}}=\left[\begin{array}{c}
0.6403 \\
-0.0123
\end{array}\right], \\
& C_{f_{11}}=\left[\begin{array}{ll}
4.3588 & -0.1614
\end{array}\right], \\
& A_{f_{12}}=\left[\begin{array}{ll}
-2.3595 & -0.9045 \\
-4.1454 & -3.4820
\end{array}\right], \quad B_{f_{12}}=\left[\begin{array}{l}
0.5199 \\
0.8936
\end{array}\right], \\
& C_{f_{12}}=\left[\begin{array}{ll}
5.5396 & 3.8431
\end{array}\right], \\
& A_{f_{21}}=\left[\begin{array}{cc}
-0.3648 & 0.0560 \\
-0.1076 & -2.8163
\end{array}\right], \quad B_{f_{21}}=\left[\begin{array}{l}
0.0161 \\
1.0228
\end{array}\right], \\
& C_{f_{21}}=\left[\begin{array}{ll}
-0.0077 & 4.4208
\end{array}\right], \\
& A_{f_{22}}=\left[\begin{array}{cc}
-3.4133 & 0.0210 \\
0.0135 & -0.5002
\end{array}\right], \quad B_{f_{22}}=\left[\begin{array}{c}
0.6410 \\
-0.0114
\end{array}\right] \text {, } \\
& C_{f_{22}}=\left[\begin{array}{ll}
4.8422 & -0.0192
\end{array}\right] \text {. }
\end{aligned}
$$

For simulation purposes, we assume the initial condition $x(0)=[0.6-0.6]^{T}$. The time delays are $\tau_{1}(t)=0.2+0.4 \sin (t), \tau_{2}(t)=0.1+0.3 \cos (t)$. The control input $u(t)$ is chosen to be $\sin (t) e^{-2 t}$. The unknown input $v(t)(t \in[030])$ is assumed to be the band-limited white noise. The fault signal $f(t)$ is simulated as a square wave signal with unit amplitude that occurred from the $10 \mathrm{~s}$ to $20 \mathrm{~s}$. Figures $1-5$ illustrate the simulation results. The possible realizations of the Markovian jumping modes of system and delay are plotted in Figures 1 and 2, respectively, where the initial modes are assumed to be $r_{0}=1$ and $s_{0}=1$. Figure 3 shows the unknown input $v(t)$. Figure 4 shows the residual signal. Figure 5 is the 


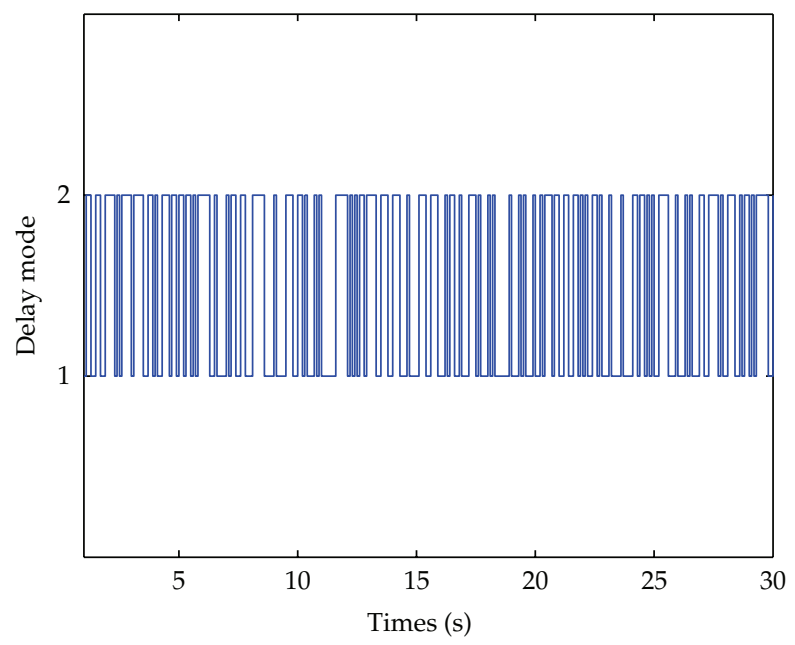

Figure 2: Delay jumping mode.

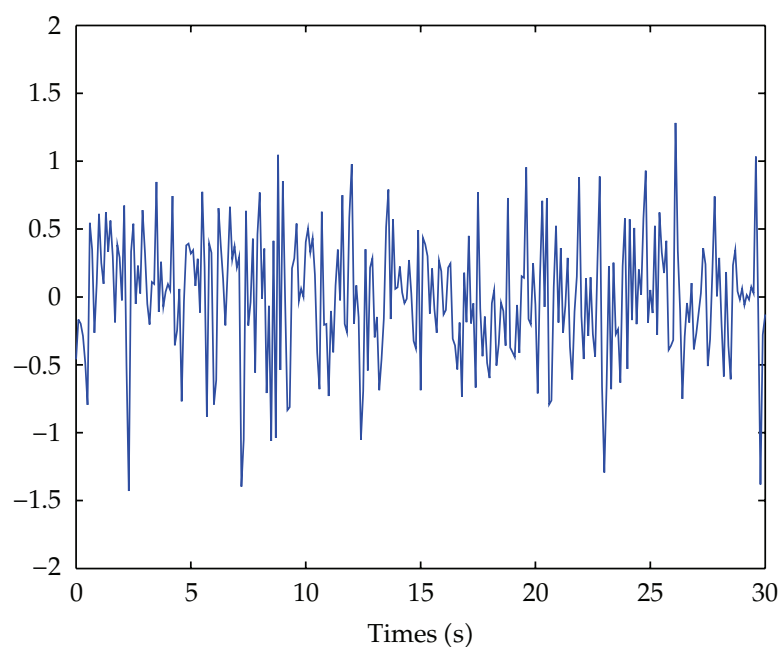

Figure 3: The unknown input $v(t)$.

simulation results of the evaluation function $f(r)$. Under the above conditions, with a selected threshold $J$ th $=0.1486$, the simulation of evaluation function $f(r)$ with fault shows that $\int_{0}^{10.9} r^{T}(t) r(t) \mathrm{d} t=0.1492>J$ th. Thus, the appeared fault can be detected after $0.9 \mathrm{~s}$. The simulation results demonstrate that the designed fault detection filter is feasible and effective.

Remark 4.2. In this study, the fault signal $f(t)$ is assumed to be a square wave signal that occurred from the $10 \mathrm{~s}$ to $20 \mathrm{~s}$. Figure 4 shows that the generated residual signal is sensitive to the fault and possesses robustness to exogenous disturbance. Furthermore, if no less than one fault appears in the systems, the designed filter is also effective to estimate fault. 


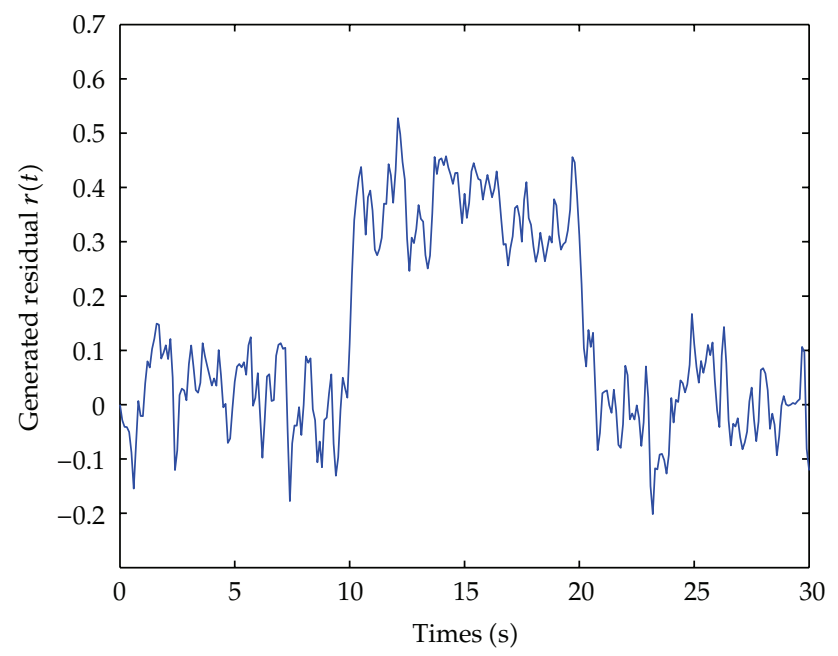

Figure 4: Generated residual signal $r(t)$.

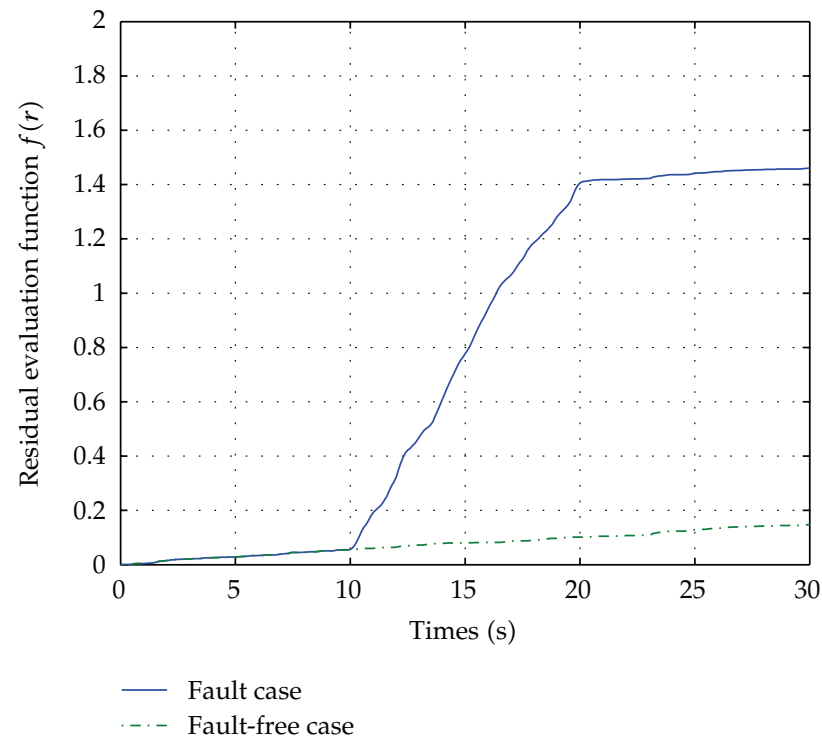

Figure 5: Evolution of residual evaluation function $f(r)$.

\section{Conclusion}

The problem of fault detection for a class of stochastic MJS is investigated in this paper. Different system mode and delay mode are considered in the model. By using the Lyapunov functional, a mixed mode-dependent sufficient condition is developed to design the stable filter. A numerical example demonstrates the effectiveness of the given method. 


\section{Acknowledgments}

This work was supported by the Fundamental Research Funds for the Central Universities (103.1.2E022050205) and the National Natural Science Foundation of China (Grant no. 61004046).

\section{References}

[1] S. X. Ding, Z. Maiying, T. Bingyong, and P. Zhang, "An LMI approach to the design of fault detection filter for time-delay LTI systems with unknown inputs," in Proceedings of the American Control Conference, pp. 2137-2142, Arlington, Va, USA, June 2001.

[2] R. H. Chen, D. L. Mingori, and J. L. Speyer, “Optimal stochastic fault detection filter," Automatica, vol. 39, no. 3, pp. 377-390, 2003.

[3] W. Li, F. Jiang, Z. Q. Wang, G. B. Zhou, and Z. C. Zhu, "Fault detection of Markov jumping linear systems," Mathematical Problems in Engineering, vol. 2012, Article ID 141867, 27 pages, 2012.

[4] X. He, Z. Wang, and D. H. Zhou, "Robust fault detection for networked systems with communication delay and data missing," Automatica, vol. 45, no. 11, pp. 2634-2639, 2009.

[5] P. Zhang and S. X. Ding, "On fault detection in linear discrete-time, periodic, and sampled-data systems," Journal of Control Science and Engineering, vol. 2008, Article ID 849546, 18 pages, 2008.

[6] C. Peng, D. Yue, E. Tian, and Z. Gu, "Observer-based fault detection for networked control systems with network Quality of Services," Applied Mathematical Modelling, vol. 34, no. 6, pp. 1653-1661, 2010.

[7] D. F. Zhang, H. Wang, B. C. Lu, and Z. Q. Wang, "LMI-based fault detection fuzzy observer design with multiple performance constraints for a class of non-linear systems: comparative study," International Journal of Innovative Computing Information and Control, vol. 8, pp. 633-645, 2012.

[8] X. M. Zhang, Z. J. Zhang, and G. P. Lu, "Fault detection for state-delay fuzzy systems subject to random communication delay," International Journal of Innovative Computing Information and Control, vol. 8, no. 4, pp. 2439-2451, 2012.

[9] M. Davoodi, H. Talebi, and H. Momeni, "A novel simultaneous fault detection and control approach based on dynamic observer," International Journal of Innovative Computing Information and Control, vol. 8, pp. 4915-4930, 2012.

[10] P. Li and V. Kadirkamanathan, "Particle filtering based likelihood ratio approach to fault diagnosis in nonlinear stochastic systems," IEEE Transactions on Systems, Man and Cybernetics C, vol. 31, no. 3, pp. 337-343, 2001.

[11] N. Meskin and K. Khorasani, "A geometric approach to fault detection and isolation of continuoustime Markovian jump linear systems," IEEE Transactions on Automatic Control, vol. 55, no. 6, pp. 1343 1357, 2010.

[12] H. R. Karimi, M. Zapateiro, and N. Luo, "A linear matrix inequality approach to robust fault detection filter design of linear systems with mixed time-varying delays and nonlinear perturbations," Journal of the Franklin Institute, vol. 347, no. 6, pp. 957-973, 2010.

[13] L. Guo and H. Wang, "Fault detection and diagnosis for general stochastic systems using B-spline expansions and nonlinear filters," IEEE Transactions on Circuits and Systems I, vol. 52, no. 8, pp. 16441652, 2005.

[14] T. Li, L. Guo, and L. Wu, "Observer-based optimal fault detection using PDFs for time-delay stochastic systems," Nonlinear Analysis: Real World Applications, vol. 9, no. 5, pp. 2337-2349, 2008.

[15] X. Mao, "Exponential stability of stochastic delay interval systems with Markovian switching," IEEE Transactions on Automatic Control, vol. 47, no. 10, pp. 1604-1612, 2002.

[16] Q. Zhu and J. Cao, "Exponential stability of stochastic neural networks with both Markovian jump parameters and mixed time delays," IEEE Transactions on Systems, Man, and Cybernetics B, vol. 41, no. 2, pp. 341-353, 2011.

[17] Y. Liu, Z. Wang, and X. Liu, "An LMI approach to stability analysis of stochastic high-order Markovian jumping neural networks with mixed time delays," Nonlinear Analysis: Hybrid Systems, vol. 2, no. 1, pp. 110-120, 2008.

[18] L. Zhang and E.-K. Boukas, "Stability and stabilization of Markovian jump linear systems with partly unknown transition probabilities," Automatica, vol. 45, no. 2, pp. 463-468, 2009.

[19] P. Shi, E.-K. Boukas, and R. K. Agarwal, "Control of Markovian jump discrete-time systems with norm bounded uncertainty and unknown delay," IEEE Transactions on Automatic Control, vol. 44, no. 11, pp. 2139-2144, 1999. 
[20] P. Shi, Y. Xia, G. P. Liu, and D. Rees, “On designing of sliding-mode control for stochastic jump systems," IEEE Transactions on Automatic Control, vol. 51, no. 1, pp. 97-103, 2006.

[21] Z. Fei, H. Gao, and P. Shi, "New results on stabilization of Markovian jump systems with time delay," Automatica, vol. 45, no. 10, pp. 2300-2306, 2009.

[22] H. Zhao, Q. Chen, and S. Xu, " $H_{\infty}$ guaranteed cost control for uncertain Markovian jump systems with mode-dependent distributed delays and input delays," Journal of the Franklin Institute, vol. 346, no. 10, pp. 945-957, 2009.

[23] Z. Wang, Y. Liu, and X. Liu, "Exponential stabilization of a class of stochastic system with Markovian jump parameters and mode-dependent mixed time-delays," IEEE Transactions on Automatic Control, vol. 55, no. 7, pp. 1656-1662, 2010.

[24] G. Wang, Q. Zhang, and V. Sreeram, "Delay-range-dependent $H_{\infty}$ control for Markovian jump systems with mode-dependent time delays," Asian Journal of Control, vol. 12, no. 6, pp. 704-713, 2010.

[25] M. Liu, P. Shi, L. Zhang, and X. Zhao, "Fault-tolerant control for nonlinear Markovian jump systems via proportional and derivative sliding mode observer technique," IEEE Transactions on Circuits and Systems I, vol. 58, no. 11, pp. 2755-2764, 2011.

[26] Z. Wu, H. Su, and J. Chu, " $H_{\infty}$ filtering for singular Markovian jump systems with time delay," International Journal of Robust and Nonlinear Control, vol. 20, no. 8, pp. 939-957, 2010.

[27] J. Liu, Z. Gu, and S. Hu, " $H_{\infty}$ filtering for Markovian jump systems with time-varying delays," International Journal of Innovative Computing, Information and Control, vol. 7, no. 3, pp. 1299-1310, 2011.

[28] Y. Dong, J. Sun, and Q. Wu, " $H_{\infty}$ filtering for a class of stochastic Markovian jump systems with impulsive effects," International Journal of Robust and Nonlinear Control, vol. 18, no. 1, pp. 1-13, 2008.

[29] H. Shen, S. Xu, X. Song, and Y. Chu, "Delay-dependent $H_{\infty}$ filtering for stochastic systems with Markovian switching and mixed mode-dependent delays," Nonlinear Analysis: Hybrid Systems, vol. 4, no. 1, pp. 122-133, 2010.

[30] H. Shao, "Delay-range-dependent robust $H^{\infty}$ filtering for uncertain stochastic systems with modedependent time delays and Markovian jump parameters," Journal of Mathematical Analysis and Applications, vol. 342, no. 2, pp. 1084-1095, 2008.

[31] H. Yan, M. Q.-H. Meng, H. Zhang, and H. Shi, "Robust $H_{\infty}$ exponential filtering for uncertain stochastic time-delay systems with Markovian switching and nonlinearities," Applied Mathematics and Computation, vol. 215, no. 12, pp. 4358-4369, 2010.

[32] C. X. Liu, Y. Q. Zhang, and H. X. Sun, "Finite-time $H_{\infty}$ filtering for singular stochastic systems," Journal of Applied Mathematics, vol. 2012, Article ID 615790, 16 pages, 2012.

[33] M. Zhong, H. Ye, P. Shi, and G. Wang, "Fault detection for Markovian jump systems," IEE Proceedings Control Theory \& Applications, vol. 152, pp. 397-402, 2005.

[34] M. Zhong, Q. Ding, and P. Shi, "Parity space-based fault detection for Markovian jump systems," International Journal of Systems Science, vol. 40, no. 4, pp. 421-428, 2009.

[35] H. Wang, C. Wang, H. Gao, and L. Wu, "An LMI approach to fault detection and isolation filter design for Markovian jump system with mode-dependent time-delays," in Proceedings of the American Control Conference, pp. 5686-5691, Minneapolis, Minn, USA, June 2006.

[36] Y. Y. Yin and P. Shi, "Gain-scheduled robust fault detectionon time-delay stochastic nonlinear systems," IEEE Transactions on Industrial Electronics, vol. 58, pp. 4908-4916, 2011.

[37] X. Yao, L. Wu, and W. X. Zheng, "Fault detection filter design for Markovian jump singular systems with intermittent measurements," IEEE Transactions on Signal Processing, vol. 59, no. 7, pp. 3099-3109, 2011.

[38] L. Zhang, E.-K. Boukas, L. Baron, and H. R. Karimi, "Fault detection for discrete-time Markov jump linear systems with partially known transition probabilities," International Journal of Control, vol. 83, no. 8, pp. 1564-1572, 2010.

[39] S. He and F. Liu, "Fuzzy model-based fault detection for Markov jump systems," International Journal of Robust and Nonlinear Control, vol. 19, no. 11, pp. 1248-1266, 2009.

[40] S. He and F. Liu, "Filtering-based robust fault detection of fuzzy jump systems," Fuzzy Sets and Systems, vol. 185, pp. 95-110, 2011.

[41] A. H. Abolmasoumi and H. R. Momeni, "Robust observer-based $H_{\infty}$ control of a Markovian jump system with different delay and system modes," International Journal of Control, Automation and Systems, vol. 9, pp. 768-776, 2011.

[42] K. Gu, "An integral inequality in the stability problem of time-delay systems," in Proceedings of the 39th IEEE Confernce on Decision and Control, pp. 2805-2810, Sydney, Australia, December 2000. 


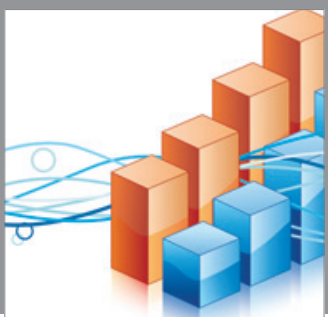

Advances in

Operations Research

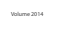

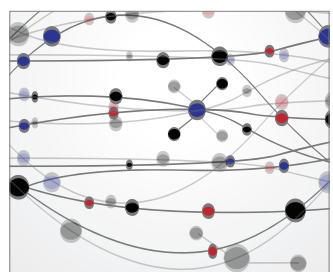

\section{The Scientific} World Journal
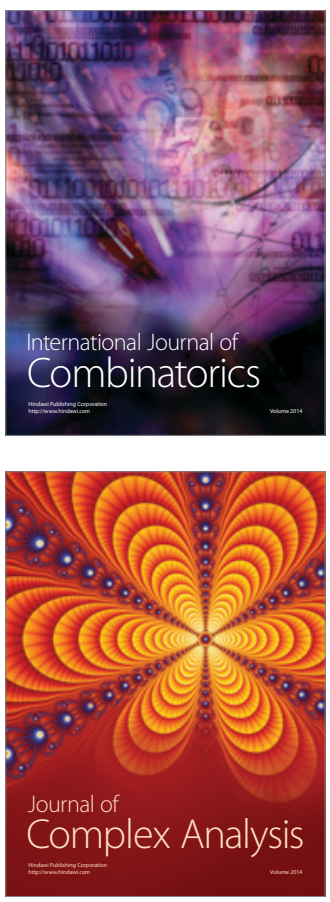

International Journal of

Mathematics and

Mathematical

Sciences
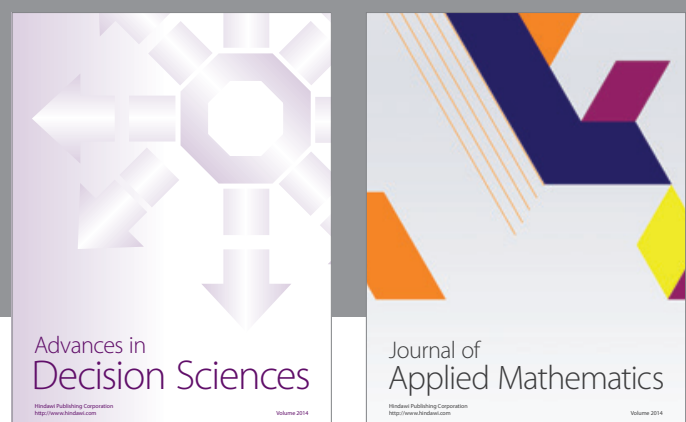

Journal of

Applied Mathematics
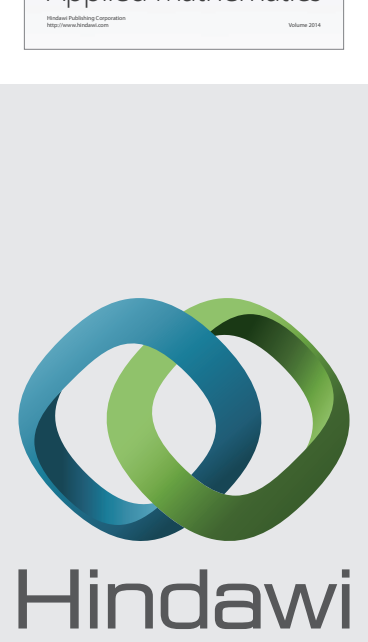

Submit your manuscripts at http://www.hindawi.com
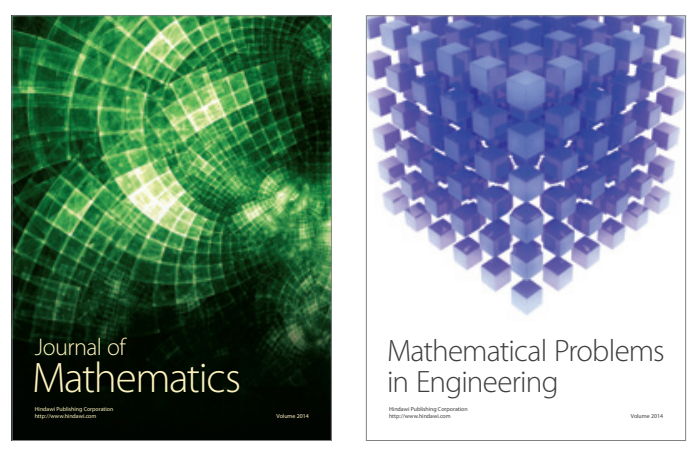

Mathematical Problems in Engineering
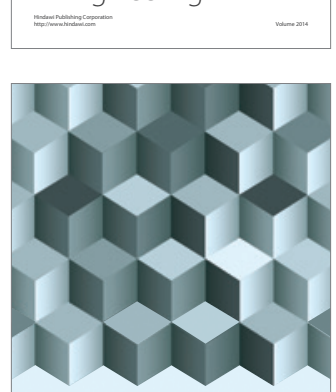

Journal of

Function Spaces
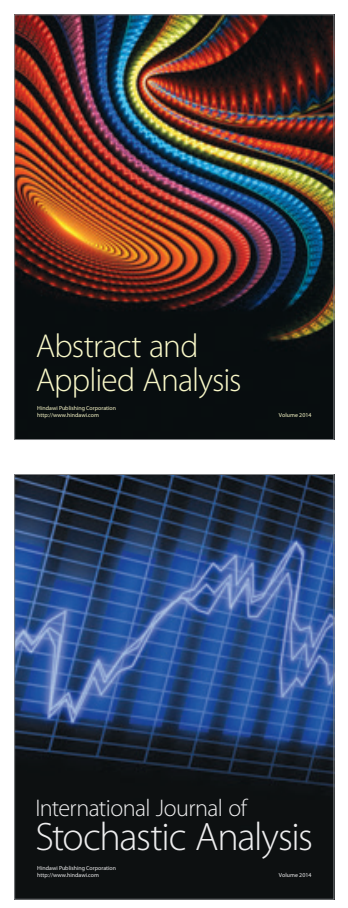

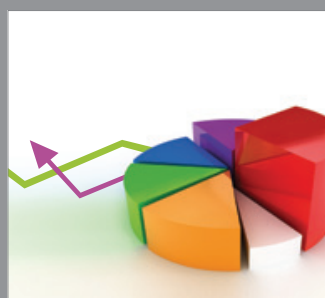

ournal of

Probability and Statistics

Promensencen
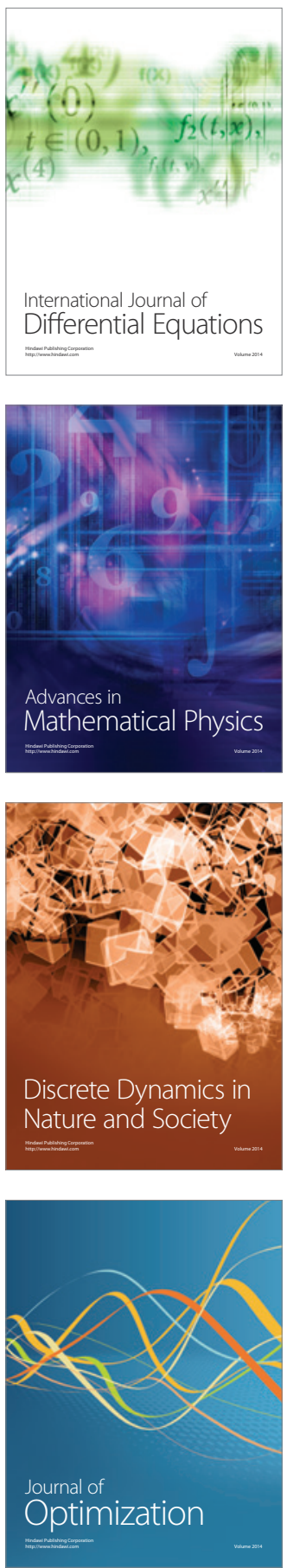